\title{
Plan de manejo para el manglar de Sontecomapan, Catemaco, Veracruz, México: una estrategia para la conservación de sus recursos naturales
}

\author{
Gustavo Carmona-Díaz ${ }^{1}$ \\ Jorge E. Morales-Mávil ${ }^{1}$ \\ Ernesto Rodríguez-Luna²
}

\begin{abstract}
RESUMEN
El manglar de Sontecomapan, ubicado en la Reserva de Biosfera Los Tuxtlas, al sur del estado Veracruz, forma parte del patrimonio natural de México y del mundo. Actualmente, se encuentra inscrito en la Lista Ramsar con el número 1342. Su importancia se reconoce en términos biológicos, culturales y económicos. Su valor biológico está fundamentado en el papel que este ecosistema representa para numerosas especies animales y vegetales, al funcionar como hábitat, refugio y zona de anidación y desove. Una característica distintiva de este manglar son las dimensiones estructurales que presentan sus especies arbóreas así como la presencia de otras plantas asociadas a este ecosistema, principalmente epífitas y orquídeas, muchas de ellas amenazadas con la extinción. Actualmente, su valor cultural está subestimado, aunque la zona del manglar de Sontecomapan tuvo relevancia desde la época prehispánica con la cultura Olmeca. A nivel económico, este manglar es fuente de alimentación e ingresos para numerosas familias. Sin embargo, presenta algunos problemas que pueden poner en riesgo su conservación, como son la tala inmoderada, el azolve, la caza furtiva y el saqueo de especies, entre otros. Este manglar constituye un área natural protegida a cargo de la Universidad Veracruzana, por lo cual, se plantea como estrategia inicial, la formulación de un Plan de manejo que intente cumplir con los objetivos de investigación, educación y conservación de este ecosistema.
\end{abstract}

PALABRAS CLAVE:

Conservación, manglar, plan de manejo, Reserva de Biosfera Los Tuxtlas, Sontecomapan.

\begin{abstract}
The Sontecomapan mangrove is located at Los Tuxtlas Biosphere Reserve, in the southern of Veracruz, Mexico. Currently, this area is listed in the Ramsar index with number 1342. Its biological, cultural and economical values are recognized. This ecosystem represents habitat, refuge, nesting area and nursery for numerous species. A distinctive characteristic of this mangrove is the high forest structure compared with other mangrove forests in Veracruz and as well as the high number of plants associated to this ecosystem, mainly epiphytes and orchids, many of them endangered. At the moment, their cultural value is underestimated, although the area of the swamp of Sontecomapan had relevance from the prehispanic time mainly by the Olmecan culture. Its economic value is as a food source and revenue for many people. However, several problems are affecting its conservation, such as the advance of human settlement on swamp land, illegal hunting, among others. This swamp constitutes a natural area protected administered by the Universidad Veracruzana. This paper described the initial strategy of preservation of this ecosystem, the formulation of a Management Plan. Investigation, education and conservation are the main objectives.
\end{abstract}

KEY WORDS:

Conservation, mangrove, forest management, Biosphere reserve Los Tuxtlas, Sontecomapan. vistas s/n. Xalapa 91000 Veracruz, México. c.e.: carmonag@ ecologia.edu.mx.

2 Universidad Veracruzana. Dirección General del Área Académica Biológico-Agropecuarias. Lomas del Estadio S/N. Colonia Zona Universitaria. Xalapa 91000 Ver., México. 


\section{INTRODUCCIÓN}

Los manglares representan un gran potencial económico y alimenticio para las poblaciones humanas que habitan en los alrededores de estos ecosistemas lagunares. Particularmente, los árboles de los manglares son apreciados como fuente de leña, carbón y madera para construcción. Sin embargo, un manejo inadecuado de estos recursos, ha hecho que los manglares estén desapareciendo a un ritmo acelerado (Tomlinson, 1986; Silva-Benavides, 1999; Villalobos et al., 1999). Se calcula que más del $40 \%$ de la extensión original del manglar en América Latina se está perdiendo por la deforestación y la sobreexplotación de los recursos pesqueros. Esto ha propiciado que en muchos países, el uso, la conservación y el manejo de sus áreas de manglar, sea uno de los puntos primordiales dentro de las administraciones gubernamentales (Lacerda y Schaeffer, 1999; Jiménez, 1999a; Lugo, 2002). Estados Unidos, Costa Rica, Panamá, Paraguay, EI Salvador, Honduras, Nicaragua, Australia, Tailandia, Nigeria, entre otros, poseen planes de manejo para el uso sostenido y la conservación de los manglares (Kjerve et al., 1997; Jiménez, 1999b; Lahman, 1999). Costa Rica es, quizá la nación donde el gobierno se preocupa más por la conservación, ya que existe un plan de manejo operativo y en pleno funcionamiento para cada área prioritaria de manglar. A este respecto, destacan por su ejecución y por los resultados favorables alcanzados, los planes de manejo del Golfo de Nicoya y de la Reserva Forestal Térraba-Sierpe. Caso contrario es el de algunos países donde no hay planes de manejo para sus manglares y, en caso de tenerlos, resultan inadecuados e inoperantes (Jiménez, 1999b; Yáñez-Arancibia y Lara-Domínguez, 1999).

En México, los manglares se presentan a lo largo de todas sus costas y constituyen uno de los ecosistemas costeros más importantes. No obstante, son pocas las áreas de manglar donde existen planes de manejo operantes que permitan su ejecución real y, por ende, la conservación de sus recursos (Valdez, 2002). En muchas otras áreas de manglar del país, es necesario establecer estos planes de manejo para evitar el continuo deterioro de los recursos naturales costeros y la pérdida gradual de estas comunidades vegetales primarias (Lugo, 2002).

Un ejemplo de esto último, lo constituye la laguna de Sontecomapan (Fig. 1), ubicada dentro de la Reserva de Biosfera Los Tuxtlas, al sur del estado de Veracruz, donde la falta de un plan de manejo propició la pérdida continua de sus recursos. El manglar de Sontecomapan, considerado como uno de los mejor conservados y menos contaminados del país, se encuentra en la localidad del mismo nombre, dentro del municipio de Catemaco y cuenta con una extensión aproximada de 891 hectáreas (Contreras y Castañeda, 1995). La superficie actual de este manglar es de aproximadamente 700 hectáreas, localizándose la mayor cobertura en el extremo noreste de la laguna (Paré et al., 1997; Ángeles, 1997; Carmona-Díaz et al., 2001).

\section{IMPORTANCIA BIOLÓGICA DEL MANGLAR DE SONTECOMAPAN}

\section{Flora}

Florísticamente, el manglar de Sontecomapan está integrado por tres de las seis especies de mangle registradas en México, las cuales son Rhizophora mangle (Rhizophoraceae) (mangle rojo), Avicennia germinans (Avicenniaceae) (mangle negro) y Laguncularia racemosa (Combretaceae) (mangle blanco). La estructura, distribución y abundancia de estas especies arbóreas confieren a este 


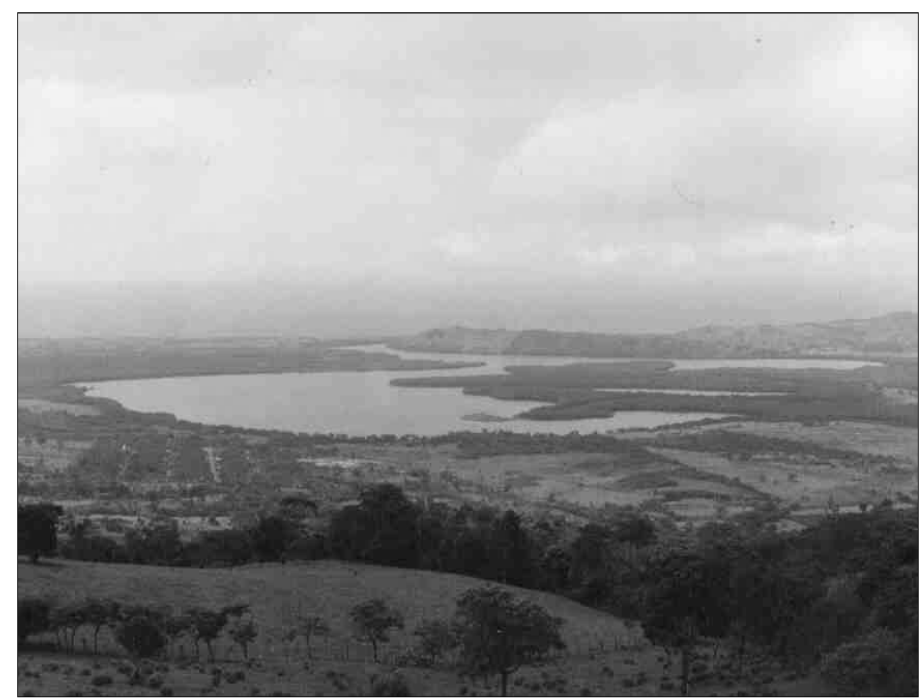

Figura 1. Panorámica del manglar de Sontecomapan, Veracruz.

manglar una gran importancia biológica; destacándose la altura de los mismos, que a veces sobrepasan los 30 metros y un diámetro a la altura del pecho superior al promedio registrado en otros manglares de México (Menéndez, 1976; Pennington y Sarukhán, 1998; YáñezArancibia y Lara-Domínguez, 1999; Carmona-Díaz y Rodríguez-Luna, 2001; López-Portillo y Ezcurra, 2002).

El manglar de Sontecomapan también funciona como hábitat para un gran número de otras formas vegetales como musgos, líquenes, helechos, epífitas no vasculares y vasculares, lianas y plantas parásitas, lo cual lo hace muy diverso, ya que generalmente, no es común encontrar otras plantas asociadas a los ecosistemas de manglar. La presencia del epifitismo vascular es una característica poco común en los manglares, no sólo en los de México sino del mundo. Los alcaloides y taninos que poseen los árboles de mangle, por lo general, no permiten el establecimiento de plantas como las epífitas. No obstante, en el manglar de Sontecomapan las epífitas están ampliamente representadas por las familias Pteridacae, Araceae, Orchidaceae, Cactaceae, Bromeliaceae, Piperaceae, Gesneriaceae y Moraceae (García-Hoyos et al., 2003).

Las orquídeas Cycnoches ventricosum (pajaritos verdes) y Chysis bractescens (flor de la Candelaria) que habitan en este manglar, se encuentran en la categorías de amenazadas (SEMARNAT, 2001); en este manglar, se ha observado que su abundancia no supera los diez ejemplares. Se conoce que estas especies permanecen cortos periodos en ciertos lugares para luego colonizar otros, mediante la dispersión de sus semillas, por lo cual necesitan grandes extensiones de bosque para establecerse y completar su ciclo biológico. El manglar de Sontecomapan presenta las características que requieren estas especies para su sobrevivencia, como son árboles hospederos con corteza rugosa, troncos en descomposición, alta humedad relativa y disponibi- 
lidad de polinizadores. En el manglar de Sontecomapan, también habitan otras especies de orquídeas, aunque, si las condiciones de saqueo y venta ilícita continúan ejerciendo presión negativa sobre éstas, muy probablemente desaparecerán de este ecosistema. Caso concreto son Brassavola nodosa (dama de noche), Encyclia alata (parásita de olor), E. belizensis (parásita guinda), Epidendrum nocturnum (dama de noche) y E. ciliare (parásita de barbas). Todas estas especies están sujetas a una fuerte presión de extracción para su venta como plantas de ornato. Cada año son extraídas grandes cantidades de ejemplares, por lo cual, en algunas áreas han sido arrasadas y su presencia está restringida a los árboles de gran altura en el interior del manglar (García-Hoyos et al., 2003). Otra especie epífita de importancia para la conservación es la bromelia Tillandsia concolor que se encuentra en peligro de extinción (SEMARNAT, 2001).

Algunas de las especies vegetales de importancia para la conservación, que habitan de forma asociada en el manglar de Sontecomapan, son los árboles Tetrorchidium rotundatum (Euphorbiaceae) (amate blanco) y Andira galeottiana (Fabaceae) (macaya). Ambas especies se encuentran en listas de especies bajo alguna categoría de riesgo. Tetrorchidium rotundatum se encuentra en la NOMECOL-059 como especie en peligro de extinción y Andira galeottiana, se encuentra catalogada por la Unión Internacional para la Conservación de la Naturaleza (UICN) como especie vulnerable muy próxima a estar amenazada de extinción (HiltonTaylor, 2000; SEMARNAT, 2001; CarmonaDíaz, 2003).

\section{Fauna}

Dentro del grupo de los peces que hacen uso de este manglar, es destacable la presencia de Lutjanus cyanopterus
(Lutjanidae) (pargo mulato), una especie que se distribuye en la costa del Atlántico desde Florida, EUA hasta Brasil, además en Las Bahamas, el Caribe y las Antillas. De acuerdo con la Fish Data Base (2003), L. cyanopterus es una especie cuyo hábitat es principalmente la zona de arrecifes y las de manglar. Se alimenta de otros peces, camarones y cangrejos. Según la UICN esta especie es considerada como vulnerable (Hilton-Taylor, 2000). En la laguna de Sontecomapan, $L$. cyanopterus ha sido recientemente registrada por primera vez (Cadena-Medel et al., 2003). La presencia de esta especie es indicadora del buen estado de conservación del sistema marino o lagunar, ya que necesita de condiciones adecuadas para sobrevivir, como la abundancia de presas, la calidad del agua y los espacios para su reproducción (Hilton-Taylor, 2000). No representa una gran demanda comercial para los pobladores, ya que sólo es capturada de manera esporádica.

Dentro de los reptiles, el cocodrilo Crocodylus moreletii (Crocodylidae), la iguana verde Iguana iguana (Iguanidae) y por lo menos cuatros especies de tortugas: la tortuga guau Staurotypus triporcatus; la taimán, Claudius angustatus (Staurotypidae); la tortuga jicotea, Trachemys scripta (Emydidae) y la pochitoque, Kinosternon leucostomun (Kinosternidae), son de las más características e importantes de la zona. Estos reptiles han sido aprovechados tradicionalmente por su carne y algunos de ellos forman parte del menú en varios restaurantes de la ciudad de Catemaco. La iguana verde, la pochitoque, la tortuga guau y la tortuga jicotea, están consideradas como especies bajo protección especial, el cocodrilo es una especie amenazada, mientras que el taimán se considera en peligro de extinción (Hilton-Taylor, 2000; SEMARNAT, 2001).

Las aves en el manglar de Sontecomapan representan un constituyente 
importante de la zona, donde pueden observarse innumerables individuos alimentándose juntos en un área reducida y representando a decenas de especies. El chorlo chiflador Charadrius melodus (Charadriidae) es una de las especies migratorias que llegan a este manglar, es característica de Norteamérica, se distribuye desde las grandes planicies del norte de Estados Unidos hasta Canadá, así como en los Grandes Lagos. Durante el invierno llega hasta la costa del Atlántico, el Golfo de México y las playas del Caribe. Según la Unión Internacional para la Conservación de la naturaleza esta especie se encuentra en peligro de extinción (Hilton-Taylor, 2000). En cuanto a las aves rapaces, el área del manglar de Sontecomapan es diversa en este grupo de animales, entre las que están la aguililla negra Buteogallus anthracinus (Accipitridae). Esta especie tiene una amplia distribución, encontrándose desde el sur de los Estados Unidos, en sus límites con México, hasta el noroeste de Perú y norte de Venezuela e isla de San Vicente en el Caribe. En Sontecomapan se puede observar como una especie residente que anida en el interior del manglar. La aguililla negra está incluida en el apéndice II de la CITES (Comisión Internacional para el Tráfico de Especies) $y$, de acuerdo con la NOM-ECOL-059 (2001), es una especie amenazada. Considerando esto, su presencia en Sontecomapan es importante para realizar estudios sobre su ecología y su conducta, así como para determinar parámetros poblacionales que ayuden a plantear estrategias para su conservación.

Otra de las especies que se presenta en el área y que tiene especial relevancia biológica, es la nutria Lontra longicaudis (Mustelidae). En México, está incluida en la lista de especies amenazadas de acuerdo con la SEMARNAT y, a nivel internacional, la UICN (Unión Internacional para la Conservación de la
Naturaleza), la considera aún con datos insuficientes (ID) para incluirla en alguna categoría de registro; la CITES, la considera dentro del Apéndice I (Hilton-Taylor, 2000). Se desconoce el estado de su población en la zona de Sontecomapan, aunque se presume bastante diezmada.

\section{PROBLEMÁTICA}

Tal como lo destaca Morales-Abril (1999), un buen diagnóstico ayudará a sentar las bases para plantear estrategias y acciones adecuadas y factibles para mitigar las fuentes y los impactos asociados a los objetos de conservación además de dar seguridad al área en el largo plazo; sin embargo, también se advierte que no en todos los casos se cuenta con información completa sobre la problemática ecológica y social del área y que ello no debe retrasar la formulación del Plan de Manejo respectivo. Aquí se presenta un diagnóstico preliminar, el cual deberá ser completado en el corto plazo, como fundamento y orientación de los programas del Plan.

La deforestación del manglar y de otros ecosistemas adyacentes a la laguna de Sontecomapan, es promovida con la finalidad de ampliar áreas para la agricultura y para la ganadería extensiva. La práctica ganadera avanza cada vez más hacia el interior del manglar, aún cuando los sitios inundables no son propicios para la cría de ganado. No obstante, los ganaderos han desarrollado técnicas para superar esta limitante, abriendo canales en los linderos de los terrenos vecinos al manglar, evitando con ello que cuando sube la marea inunde los campos ganaderos.

Así mismo, las prácticas agrícolas que se realizan en la zona, están sustituyendo manchones de selva para introducir la agricultura de temporal. Por ejemplo, los cultivos tradicionales de maíz 
y frijol son reemplazados por el de chile, hoy en día generador de empleos para los habitantes de la región. Esto también ha ocasionado graves problemas ambientales como son el azolve de los ríos y la laguna por arrastre eólico y fluvial, lo cual impide la circulación normal de lanchas turísticas y de pescadores; descenso de los niveles de profundidad de los ríos y la laguna; cambios de temperatura en los cuerpos de agua; y es causa de descenso de los niveles de productividad pesquera en el mismo y contaminación de los ríos y de la laguna de Sontecomapan por el vertido de químicos como fertilizantes, insecticidas y herbicidas.

Otro problema crítico que se observa en el área de Sontecomapan, es la extracción ilegal de plantas y animales silvestres, así como el uso inadecuado de los recursos forestales y pesqueros. Entre las plantas extraídas del manglar con fines ornamentales se encuentran las orquídeas, las bromelias y las cactáceas. Los árboles de mangle son descortezados por los pobladores que se dedican a la curtiduría, lo cual causa una gran mortandad de individuos, ya que generalmente, el descortezamiento del árbol se realiza en toda su circunferencia; esto impide el ascenso de la savia y los nutrientes, actividad conocida en la zona como "ligamiento", misma que ocasiona la muerte del árbol. De la fauna silvestre, destaca el caso de los cotorros y pericos que anidan en los árboles de mangle y que son capturados para su venta como mascotas.

Existe conflicto por la captura de especies acuáticas ya que, aunque regularmente se distribuyen circulares informativas donde se mencionan los periodos de veda para algunas especies, por lo regular éstos no se cumplen. Muchos pescadores aprovechan que al disminuir la oferta aumenta la demanda del producto; así, ellos obtienen buenas ganancias aunque sólo sean temporales.
El tráfico de lanchas también es otro problema en el área, ocasiona un aumento en el oleaje provocando desbordamientos en las orillas y contribuye al azolve de los ríos y de la propia laguna.

La cacería dentro del manglar de Sontecomapan se presenta sólo esporádicamente y está dirigida hacia aquellas especies que son muy abundantes como los mapaches. Sin embargo, existe una cacería de tipo oportunista cuando son cazadas aquellas especies que son avistadas por los lugareños al efectuar sus actividades diarias; tal es el caso de la nutria, el cocodrilo y la boa, entre otras (Morales-Mávil y Villa-Cañedo, 1998).

Frente a la problemática ambiental que presenta el manglar de Sontecomapan y con la finalidad de conservar y manejar los recursos naturales que ofrece esta zona, se ha propuesto que el manglar de Sontecomapan sea reconocido como un Área Natural Protegida.

\section{ESTRATEGIA DE CONSERVACIÓN}

En noviembre de 1998, la región de Los Tuxtlas fue decretada Reserva de la Biosfera; la Laguna de Sontecomapan está incluida en ella. Sin embargo, de acuerdo con la zonificación de esta reserva, tanto la laguna de Sontecomapan como su manglar, quedaron ubicados en la zona de amortiguamiento, con lo cual, este sistema lagunar quedó expuesto al continuo y progresivo deterioro ambiental que, en general, está sufriendo la región.

Por todo lo anterior y por las particularidades ecológicas de esta comunidad vegetal, en 1999, el Patronato ProUniversidad Veracruzana, A. C., propuso reconocer al Manglar de Sontecomapan como Área Natural Protegida, con la finalidad principal de conservar el ecosis- 
tema. Para tal efecto, la Secretaría de Medio Ambiente y Recursos Naturales (SEMARNAT), emitió el 22 de junio de 2000, un título de concesión (No. DGZF087/00) en el que autoriza al Patronato Pro-Universidad Veracruzana A.C., para ocupar y aprovechar $3751415 \mathrm{~m}^{2}$ (casi 400 hectáreas) de zona federal marítimaterrestre, de acuerdo con el polígono referido en el título. Esta concesión tiene una vigencia de 25 años y puede ser prorrogable en los términos que establece la Ley General de Bienes Nacionales. Es por ello que se planteó estructurar y operar un Plan de Manejo del manglar de Sontecomapan, que se propone cumplir con los objetivos de investigación, conservación y educación. Esta concesión permite al Patronato la realización de tareas conducentes a la protección, conservación ecológica e investigación científica. La realización y ejecución del Plan de Manejo quedará a cargo de la Universidad Veracruzana dentro del marco normativo que establece el Plan de Manejo de la Reserva de la Biosfera Los Tuxtlas y las regulaciones estatales y federales de orden superior.

\section{OBJETOS DE CONSERVACIÓN}

De acuerdo con la información presentada, se han identificado como parte fundamental de este Plan de Manejo los objetos de conservación las comunidades vegetales de manglar, las especies indicadoras y los procesos biológicos.

- El manglar

- Las orquídeas Cycnoches ventricosum y Chysis bractescens

- Los árboles Tetrorchidium rotundatum y Andira galeottiana

- El pez Lutjanus cyanopterus

- El gremio de tortugas

- La iguana verde (Iguana iguana)

- El cocodrilo (Crocodylus moreletii)

- La comunidad de aves playeras
- La aguililla negra (Buteogallus anthracinus)

- La nutria (Lontra longicaudis)

Con base en los objetos de conservación se plantearon las siguientes metas:

- Cuidar la persistencia del manglar.

- Mantener las poblaciones de orquídeas, iguana verde, cocodrilo, tortuga taimán, aguililla negra y nutria en el manglar.

- Estudiar, proteger y mejorar las condiciones ambientales que requiere el gremio de tortugas.

- Estimar el estado de la población de tortuga taimán, cocodrilo, iguana verde, aguililla negra y nutria.

- Mantener las condiciones adecuadas del ecosistema para que las aves migratorias playeras se refugien en el área.

Este Plan de manejo debe fundamentar y orientar las acciones que aseguren la permanencia del ecosistema manglar en la Laguna de Sontecomapan así como aportar una guía para el uso sostenido de los recursos naturales que le son propios y relativos. Para tal fin, este Plan cumplirá los siguientes objetivos:

- Aportar una descripción de la situación actual del manglar y de los factores que le afectan.

- Proponer un esquema y lineamientos para desarrollar un programa de investigaciones biológicas básicas encaminadas a incrementar el conocimiento de los recursos naturales de la zona y a fundamentar una estrategia para su conservación y aprovechamiento.

- Identificar, eliminar y/o reducir los problemas básicos que están afectando la permanencia del ecosistema manglar. Particularmente, inferir sobre la tala indiscriminada, el uso del suelo, el azolve, la extracción de recursos, la 
contaminación y el efecto de borde.

- Constituir un cuerpo de conocimientos que dé sustento a la educación ambiental en pro de la conservación del manglar.

- Aportar un documento base como fundamento para otros proyectos en materia de investigación, conservación y educación.

- Sumar esfuerzos conservacionistas dentro de la Reserva de Biosfera Los Tuxtlas, incorporando al manglar como ecosistema protegido.

- Influir en la regulación de la actividad turística, con la finalidad de reducir la perturbación sobre las comunidades bióticas. Proponer rutas ecoturísticas.

Con este plan de manejo para la conservación del manglar de Sontecomapan, la Universidad Veracruzana, pretende dar comienzo a una estrategia global de conservación en pro de los manglares veracruzanos.

\section{PROGRAMAS Y ESTRATEGIAS}

Los programas y estrategias de manejo serán los medios que permitirán alcanzar los objetivos de conservación del manglar de Sontecomapan. Dentro de estas estrategias se deberán organizar y coordinar acciones con habitantes locales, dependencias de gobierno e instituciones académicas para establecer y ejecutar acuerdos de cooperación.

Considerando los objetivos de conservación y las metas establecidas, así como la problemática identificada en el área, se plantean los siguientes objetivos y acciones mediante tres programas básicos: Investigación, Educación y Conservación.

\section{Investigación}

Objetivos:

- Elaborar un listado completo de las especies de flora y fauna acuáticas y terrestres del manglar.

- Determinar parámetros poblacionales de las especies consideradas objetos de conservación.

- Caracterizar el área con base en la distribución de las especies de mangle y grados de perturbación.

- Describir la herbivoría sobre las especies de mangles.

- Determinar patrones fenológicos de las especies arbóreas.

- Identificar el uso que los habitantes de la región hacen de los recursos naturales locales (pesca, caza, cultivos y recolección, entre otros).

- Generar información que haga posible la elaboración de proyectos productivos que brinden beneficios a la gente de las comunidades locales.

- Crear y alimentar una base de datos con información sobre los recursos del manglar de Sontecomapan.

- Establecer programas permanentes de evaluación de poblaciones y de aprovechamiento de recursos florísticos y faunísticos.

El manglar de la Laguna de Sontecomapan ha recibido poca atención por parte de investigadores, por lo cual existe desconocimiento acerca de los elementos bióticos que lo integran, la forma en que están estructurados y cuál es la dinámica que los rige. En esta perspectiva, la conservación del manglar dependerá, en gran medida, del manejo de los recursos de la zona, lo cual deberá basarse en la investigación.

La mayor parte de la investigación básica que se ha realizado en la zona se 
ha centrado en la ictiofauna e invertebrados asociados a los cuerpos de agua. Poco se sabe de la flora y fauna terrestres que habitan el ecosistema del manglar, por ello, el listado de las especies, su distribución y abundancia, las interacciones ecológicas y la determinación del estado de conservación, deberán ser tareas prioritarias dentro de las actividades de monitoreo de la biodiversidad.

La investigación científica es indispensable para comprender ecológicamente este sistema biótico y así, lograr su conservación. La información que se genere, permitirá conocer no sólo la riqueza biológica con que cuenta este lugar, sino también los aspectos que la determinan, así como las relaciones que existen entre los componentes bióticos y abióticos del mismo. Esto ayudará a precisar la situación actual del área y su problemática, de tal manera que se cuente con los fundamentos para dirigir alternativas de conservación, restauración y manejo de recursos.

Será necesario operar varias estaciones de muestreo en el área para dirigir las actividades de investigación básica, de las cuales, puedan derivarse elementos para la realización de investigación aplicada y así generar una base de datos como soporte para otras líneas y proyectos de investigación.

Las investigaciones en el área estarán reguladas por un reglamento bajo la supervisión del Director del Área y del Jefe de Programa.

Las prioridades de investigación estarán dirigidas hacia el conocimiento de la flora y fauna presentes en la zona. Dentro de éstas serán, estudiar las poblaciones de aves identificando especies residentes y migratorias, de peces, crus- táceos y moluscos para llevar a cabo estudios autoecológicos con las especies identificadas con algún grado de amenaza y realizar investigación de tipo sinecológica. Esto ayudará a obtener información relevante sobre poblaciones y comunidades, interacciones plantaanimal y migraciones.

Las investigaciones se enfatizarán hacia los objetos de conservación identificados, como son las especies de mangle, la comunidad de aves playeras y aquellas especies con algún grado de amenaza o endémicas, como algunos árboles y orquídeas, las tortugas, el cocodrilo, la iguana verde, el aguililla negra, el chorlito chiflador y la nutria, pero con una visión integral como elementos del ecosistema.

Además, parte de la investigación estará enfocada al conocimiento del uso tradicional y aprovechamiento de los recursos, para evaluar su impacto por la comunidad de Sontecomapan y las actividades ecoturísticas; así como la sucesión ecológica de las áreas desmontadas o deforestadas y que son ocupadas para actividades productivas mediante técnicas de recuperación de suelos.

Para avanzar en las labores de investigación, será necesario fomentar el trabajo conjunto entre organizaciones gubernamentales, no gubernamentales, educativas y de investigación, con la finalidad de plantear proyectos y realizar acciones que tiendan a generar conocimiento a corto, mediano y largo plazo, que permita la conservación y el manejo sostenido de los recursos del área. Además de gestionar apoyos financieros para investigación como becas para estudiantes e investigadores. En la tabla 1 se sintetizan la acciones de investigación propuestas. 
Tabla 1. Acciones de investigación propuestas a corto, mediano y largo plazo para el manejo del manglar de Sontecomapan, Catemaco, Ver

ACCIONES

CORTO MEDIANO LARGO

1-5 años 5-10 años 10-25 años

Elaborar un inventario de los recursos naturales

existentes en el área. Particularmente realizar

inventarios florísticos y faunísticos de las especies

presentes en el Manglar de Sontecomapan,

$\mathrm{X} \quad \mathrm{X}$

identificando aquellas que se encuentren bajo algún

estatus de protección (SEMARNAT o UICN) o que

tengan importancia económica.

Conocer los factores físicos, químicos y ambientales

que forman parte integral del manglar, así como la

dinámica que los rige.

Realizar un inventario de las actividades productivas que se realizan en el manglar de Sontecomapan y estimar sus impactos sobre ecosistemas y especies.

Desarrollar estudios para determinar los factores que condicionan la distribución, abundancia y estado de las especies objetos de conservación.

$x \quad x$

Monitorear las poblaciones de flora y fauna nativas del manglar con especial énfasis en las condiciones ecológicas prevalecientes.

\section{$\mathrm{X}$}

Generar información sobre las poblaciones y comunidades, las interacciones planta-animal (polinización, dispersión de semillas y herbivoría, entre

$x \quad x \quad x$
otras) y las migraciones.

Realizar investigaciones de tipo autoecológico y sinecológico con las especies identificadas con algún grado de amenaza o bajo algún estatus de protección.

$x \quad x \quad x$

Determinar las tasas de aprovechamiento de las especies identificadas con potencial económico o comercial.

$x \quad x \quad x$

Obtener información acerca del manejo, uso tradicional y aprovechamiento de los recursos naturales, por parte de los habitantes de la localidad. 
Tabla 1 (cont.). Acciones de investigación propuestas a corto, mediano y largo plazo para el manejo del manglar de Sontecomapan, Catemaco, Ver

ACCIONES

CORTO MEDIANO LARGO

1-5 años 5-10 años 10-25 años

Impulsar la investigación sobre el impacto del uso y aprovechamiento de los recursos naturales por parte de la población local.

$X \quad X$

Integrar la información sobre la sucesión ecológica en áreas deforestadas y sobre la aplicación de técnicas para recuperación de suelos y del manglar.

$X \quad X$

Mantener una base de datos sobre las investigaciones desarrolladas y en curso en el manglar de Sontecomapan para su divulgación, así como un $X$ acervo bibliográfico especializado.

Actualizar la información cartográfica del manglar de Sontecomapan en un sistema de información geográfica.

Establecer un sistema para el seguimiento de los tres programas del Plan.

$\mathrm{X} \quad \mathrm{X}$

Fomentar el trabajo en conjunto entre organizaciones gubernamentales, no gubernamentales, educativas y de investigación.

Gestionar apoyos financieros para el desarrollo de la investigación y becas para la formación de estudiantes e investigadores en el manglar de Sontecomapan.

\section{$x$}

Ayudar a la integración organizada de los grupos humanos residentes en la zona

Formar y capacitar a estudiantes y personas interesadas en integrarse a las labores de manejo $y \quad X$ conservación del manglar.

Establecer talleres demostrativos de uso sustentable de recursos, dirigidos principalmente a grupos y personas de las comunidades aledañas al área $x \quad x \quad x$ protegida 


\section{Educación}

Objetivos:

- Diseñar y ejecutar un programa educativo para la concientización hacia la problemática ambiental de la zona.

- Elaborar e impartir cursos de capacitación y talleres educativos en relación al aprovechamiento de los recursos del manglar, a todos los niveles y sectores de la población local.

- Coordinar actividades de educación formal y no formal para reforzar los programas educativos locales y regionales.

- Integrar a miembros de las comunidades local y regional en las actividades de educación y difusión que se desarrollen.

- Involucrar a los medios de comunicación locales, regionales y estatales (prensa, radio y televisión) en el programa educativo.

- Insertar experiencias educativas en el programa de investigación.

- Desarrollar, mediante ecoturismo, actividades recreativas y culturales con miras a difundir la importancia del manglar y la problemática que enfrenta.

- Fomentar la creación de asociaciones civiles para el cuidado ambiental, principalmente con estudiantes y personas de la región.

- Gestionar apoyos gubernamentales y no gubernamentales para llevar a cabo actividades de difusión en pro del manglar.

Los problemas de deforestación, contaminación y extracción de recursos que afectan al manglar de Sontecomapan, no se podrán revertir si no hay una transformación en el conocimiento, los valores y la conducta de los pobladores de la región. La educación debe ser una estrategia fundamental de apoyo a los trabajos de conservación e investigación del área.
Los planes de estudios en Sontecomapan, tanto a nivel primaria como secundaria, no ofrecen información acorde a la problemática ambiental regional. Esto determina que exista un lamentable desconocimiento local acerca de las causas, efectos y soluciones hacia los problemas regionales. Por ello, se requiere estructurar un programa que involucre proyectos y actividades de educación formal y no formal.

a) Subprograma Educación formal. Dentro de este tipo de educación a nivel universitario, se pretende el desarrollo de prácticas escolares y cursos para estudiantes de licenciatura y de posgrado, cursos de actualización académica, talleres de capacitación profesional, actividades para prestadores de servicio social y realización de tesis. Todo esto en articulación con el sector educativo superior, principalmente con las propias entidades académicas de la Universidad Veracruzana.

Parte fundamental de este apartado educativo será dirigir la atención hacia los pobladores regionales, con la finalidad de que se involucren en los proyectos de capacitación sobre las pautas de manejo eficiente de los recursos y conservación de la biodiversidad, así como en la promoción de los valores del manglar y de los humedales en general. Esto ayudará a crear o, en todo caso, a incrementar una conciencia de valoración acerca de la importancia ecológica del manglar facilitando la integración voluntaria de los pobladores hacia los programas de conservación de la zona.

\section{b) Subprograma Educación no formal.} En este rubro, se estructurará una propuesta educativa ambiental, tanto a nivel local como regional, dirigida principalmente a motivar la reflexión y el análisis de problemas particulares, de tal modo que puedan consolidarse acciones ambientales. Este subprograma educa- 
tivo, se enfocará a diferentes niveles y sectores: hacia población infantil, amas de casa, pescadores, agricultores, ganaderos, prestadores de servicios y visitantes turísticos. Con esto, se pretende buscar un cambio de actitud hacia el ambiente a través de la sensibilización y concientización individual y grupal. Se destacarán las relaciones sociedad-naturaleza y los principales problemas que afronta la población local de Sontecomapan. Se proponen anuncios en la radio, reuniones abiertas con los pescadores en las cooperativas, con los agricultores en la asamblea ejidal, con las amas de casa en sus hogares y con los ganaderos en su asociación local.

Será necesario promover una conciencia conservacionista en los turistas por medio de folletos, con la venta de playeras, calcomanías y otros objetos que los mismos pobladores locales podrán comercializar para obtener ayuda para la conservación del manglar. Dentro de las diversas estrategias educativas para dirigir mensajes sobre problemas y soluciones ambientales, se considerarán talleres, teatro guiñol y pláticas para niños de educación preescolar, videos, folletos y visitas guiadas al manglar, para alumnos de nivel primaria y bachillerato. Los estudiantes universitarios de biología y de otras carreras afines, principalmente de la Universidad Veracruzana así como de otras instituciones educativas, podrán apoyar en el programa educativo, a fin de promover la participación y concientización de la gente para colaborar en las tareas para promover la conservación del manglar y el uso racional de recursos. La participación de organizaciones civiles no gubernamentales será fundamental para agilizar el cumplimiento de estas actividades.

Será importante gestionar el apoyo gubernamental e institucional para llevar a cabo actividades de difusión en defensa del ambiente. Dentro de los temas priori- tarios, se deberá destacar la importancia ecológica para mantener la biota regional, fomentar la clasificación y el reciclaje de desechos y la necesidad de mantener la calidad del agua.

La información generada en los subprogramas, deberá ser difundida a través de la publicación de artículos científicos y divulgativos en revistas, libros y prensa escrita. En la tabla 2 se presentan las acciones propuestas para educación.

\section{Conservación}

Objetivos:

- Proponer una zonificación para el desarrollo de las actividades en el área protegida de manglar.

- Identificar y clasificar las zonas del manglar con mayor perturbación, para desarrollar actividades de mantenimiento y restauración.

- Gestionar ante las dependencias municipales, estatales y/o federales para que se cumpla la regulación del uso de la madera de mangle.

- Crear un sistema efectivo de vigilancia e inspección del sistema lagunar.

- Promover el cambio de la actividad turística al ecoturismo en la zona, como una alternativa productiva que ayude a la conservación.

- Establecer actividades de difusión y apreciación de la naturaleza.

- Colaborar con el municipio para establecer mecanismos de colecta de basura en las zonas de influencia.

- Monitorear la calidad del agua y el uso del suelo en zonas aledañas y de influencia sobre el ecosistema.

- Desarrollar programas piloto sobre uso sostenido de recursos.

- Gestionar apoyos financieros para el desarrollo de actividades de conservación en la zona.

- Coordinar acciones conjuntas con los programas de investigación y educación propuestos. 
Tabla 2. Acciones de educación propuestas a corto, mediano y largo plazo para el manejo del manglar de Sontecomapan, Catemaco, Ver.

\begin{tabular}{|c|c|c|c|}
\hline ACCIONES & $\begin{array}{l}\text { CORTO } \\
1-3 \text { años }\end{array}$ & $\begin{array}{l}\text { MEDIANO } \\
\text { 3-5 años }\end{array}$ & $\begin{array}{l}\text { LARGO } \\
\text { 5-10 años }\end{array}$ \\
\hline $\begin{array}{l}\text { Desplegar una campaña local de información y } \\
\text { educación donde se enfatice la problemática de la } \\
\text { tala del mangle, la contaminación del agua y el } \\
\text { saqueo de la flora y la fauna. }\end{array}$ & $X$ & & \\
\hline $\begin{array}{l}\text { Impartir talleres en los planteles educativos de la } \\
\text { localidad, mediante los que se brinde información } \\
\text { sobre los valores, manejo y protección de los } \\
\text { recursos naturales del manglar de Sontecomapan. }\end{array}$ & $X$ & $X$ & $X$ \\
\hline $\begin{array}{l}\text { Elaborar trípticos donde se dé a conocer la } \\
\text { importancia del manglar y los problemas que } \\
\text { puedan afectarlo con sus consecuencias. }\end{array}$ & $X$ & $X$ & \\
\hline $\begin{array}{l}\text { Diseñar materiales educativos dirigidos al turismo, } \\
\text { en los que se destaque la importancia cultural del } \\
\text { manglar, enfatizando la necesidad de su } \\
\text { conservación. }\end{array}$ & $X$ & $X$ & $X$ \\
\hline $\begin{array}{l}\text { Establecer los acuerdos de colaboración con los } \\
\text { medios de comunicación locales, regionales y } \\
\text { estatales, para difundir la gestión del manglar de } \\
\text { Sontecomapan. }\end{array}$ & $X$ & $X$ & $X$ \\
\hline $\begin{array}{l}\text { Apoyar y asesorar a los emprendedores locales } \\
\text { interesados en desarrollar actividades } \\
\text { ecoturísticas. }\end{array}$ & $X$ & $X$ & $X$ \\
\hline $\begin{array}{l}\text { Publicar un libro sobre la historia natural del } \\
\text { manglar de Sontecomapan, como fuente de } \\
\text { información e inspiración para otras tareas } \\
\text { educativas. }\end{array}$ & $X$ & $X$ & $x$ \\
\hline $\begin{array}{l}\text { Ofrecer prácticas escolares y cursos cortos, a } \\
\text { profesores y estudiantes universitarios } \\
\text { interesados en temas biológicos y en el uso y } \\
\text { conservación de los recursos naturales. }\end{array}$ & $X$ & $X$ & $X$ \\
\hline $\begin{array}{l}\text { Brindar cursos de actualización para manejadores } \\
\text { de Áreas Naturales Protegidas y de vida silvestre. }\end{array}$ & $X$ & $X$ & $X$ \\
\hline $\begin{array}{l}\text { Constituir la base para el establecimiento de dos } \\
\text { carreras de Técnico Superior Universitario; una } \\
\text { sobre Manejo de Vida Silvestre y otra sobre } \\
\text { Ecoturismo. }\end{array}$ & $X$ & $X$ & $X$ \\
\hline
\end{tabular}


De acuerdo con la información que se genere, se deberá hacer una categorización preliminar de la zona, identificando aquellas áreas que sufren mayor impacto humano y pérdida de biodiversidad. Se deberán dirigir esfuerzos hacia la restauración ecológica de esas áreas, aminorando o desapareciendo las causas que están originando esa afectación y, al mismo tiempo, desarrollando programas de reforestación, reintroducción y repoblación de especies en los casos necesarios.

Los habitantes de la zona de Sontecomapan extraen algunos productos del manglar. Por ejemplo, utilizan madera para leña, varas para pesca, troncos para durmientes y vigas, cercas para potreros y ramas y hojas por sus propiedades analgésicas y desinfectantes. El uso de alguna de las partes de los árboles, de ninguna manera ponen en riesgo la permanencia del manglar; sin embargo, la tala de individuos o de una parte importante de estos, causa una alteración drástica en este frágil ecosistema, de tal manera que deberá considerarse un programa adecuado que planifique y ordene el desarrollo de estas actividades.

Para lograr lo anterior, será necesario el apoyo de las dependencias municipales, estatales y federales para que se promueva una regulación del uso de los recursos del manglar. En particular, la extracción de madera y su afectación al manglar, sería el punto principal para gestionar esta regulación.

Otra de las estrategias básicas para asegurar que los proyectos que se establezcan se desarrollen exitosamente, será la de lograr vigilancia e inspección adecuadas. Para ello, se requiere gestionar ante las autoridades correspondientes el apoyo para llevar a cabo estas actividades.
Es deseable involucrar a la sociedad civil, principalmente a personas de la localidad, en las tareas de vigilancia, a través de incentivos económicos y programas de manejo sostenido de recursos, donde ellos encuentren un beneficio por realizar esa tarea.

Lograr que exista vigilancia en el área, ayudará a resolver muchos de los problemas generados por la influencia humana, tales como, tala, cacería furtiva y pesca de especies sin talla comercial y en periodos de veda, así como la generalizada extracción de flora y fauna. Los programas de investigación y educación, también se realizarían con mayor seguridad de éxito, de tal manera que se pueda asegurar la conservación del ecosistema y sus procesos.

El turismo es una actividad común en la zona, sin embargo, la falta de control y planeación sobre ésta, ha provocado conflictos entre lancheros, irregularidad en los ingresos (temporadas altas y otras muy bajas) y, sobre todo, alteración en el paisaje y afectación en poblaciones y comunidades faunísticas. Ante esta situación, será necesario proponer rutas turísticas que sean de bajo riesgo para la fauna. Además, una alternativa importante puede ser promover el turismo ecológico, ya que el paisaje que conjunta la vegetación, la laguna y el mar, aunado a la gran diversidad biológica de la zona, dan la posibilidad de plantear y desarrollar esta alternativa. Con una buena orientación, el ecoturismo puede rendir importantes ganancias económicas a los pobladores locales. Actualmente, esta actividad sólo rinde beneficios en temporadas cortas, particularmente en periodos vacacionales. Sin embargo, con el turismo ecológico se pueden plantear programas que permitan tener un desarrollo económico mucho más amplio, donde se realice 
una interacción del visitante con el ambiente, enfatizando la necesidad de la conservación del ambiente. Dentro de esta actividad, además de los recorridos por el manglar, los pobladores podrían realizar diferentes tareas como: participar en venta de artesanías, colaborando en campamentos o como guías turísticos.

Otro de los problemas que están afectando el área, es el incremento de desechos sólidos, una parte de los cuales es depositada directamente en el sitio y otra es acarreada por las corrientes de agua. Esto provoca que en varias partes de la laguna se concentren una gran cantidad de desechos que afectan el paisaje y pueden estar influyendo negativamente en las comunidades acuáticas. Por ello, se hace necesario retirar los desechos que ya existen e intentar eliminar los factores que están causando este fenómeno. Inicialmente se debe gestionar ante el municipio, un sistema periódico de colecta de desechos, que ayude a aminorarlos; además de considerar dentro del programa educativo, su posible manejo y el reciclaje de desechos.

Parte fundamental para el mantenimiento del ecosistema, es la calidad del agua. No sólo la biota acuática depende de esto, sino que gran parte de los organismos terrestres se ven afectados por cambios en la salinidad, en la turbidez y en la contaminación química y orgánica de los cuerpos de agua.

Es indispensable mantener un registro constante de la calidad del agua, para prevenir posibles afecciones a la biota del lugar. La coordinación con los gobiernos federales, estatales y municipales, podrían ayudar a que este monitoreo se realice exitosamente. Las acciones de conservación propuestas se presentan el la tabla 3 .

El Plan de manejo es el resultado de una primera fase metodológica, de acuerdo con la propuesta de planificación de áreas naturales protegidas realizada por The Nature Conservancy (MoralesAbril, et al., 1999). Esta fase se denomina Formulación, siendo el inicio del esfuerzo estratégico para planear el estudio, la conservación y el manejo de una de las zonas de manglar más importantes del estado de Veracruz. A partir de aquí se desarrollarán las siguientes fases metodológicas que incluyen básicamente la operación y evaluación de las actividades realizadas. Estas fases son: Instrumentación (elaboración y ejecución de los programas operativos anuales), Control y Seguimiento (presentación de informes técnicos y financieros) y Evaluación (evaluación anual de cumplimiento y éxito).

\section{REFERENCIAS}

Ángeles, G. 1997. Historia natural de las especies: Rhizophora mangle. In: González, E.; R. Dirzo y R.C. Vogt, (eds) Historia Natural de Los Tuxtlas. Universidad Nacional Autónoma de México. México, D.F. p:148-149.

Cadena-Medel, L.; G. Carmona-Díaz y E. Rodríguez. 2003. Diversidad, abundancia y distribución de la ictiofauna en la laguna de Sontecomapan, Catemaco, Veracruz, México. Mesoamericana 7(1):18-19.

Carmona-Díaz, G. y E. Rodríguez-Luna. 2001. Estructura, distribución y abundancia del manglar de Sontecomapan, Catemaco, Veracruz. Memorias del XXI Congreso Mexicano de Botánica. Querétaro, Querétaro. 118 p.

Carmona-Díaz, G. 2003. Rescate de especies de flora en el nuevo camino de acceso a la refinería de Minatitlán, Veracruz, México. PEMEX 
Tabla 3. Acciones de conservación propuestas a corto, mediano y largo plazo para el manejo del manglar de Sontecomapan, Catemaco, Ver.

\begin{tabular}{|c|c|c|c|}
\hline ACCIONES & $\begin{array}{l}\text { CORTO } \\
1-2 \text { años }\end{array}$ & $\begin{array}{l}\text { MEDIANO } \\
2-5 \text { años }\end{array}$ & $\begin{array}{l}\text { LARGO } \\
\text { 5-10 años }\end{array}$ \\
\hline $\begin{array}{l}\text { Localizar los sitios deforestados para llevar a cabo } \\
\text { actividades de restauración en los lugares con mayor } \\
\text { grado de perturbación. }\end{array}$ & $X$ & $X$ & \\
\hline $\begin{array}{l}\text { Gestionar ante las diferentes instancias municipales, } \\
\text { federales y estatales, la regulación en la explotación } \\
\text { de madera del mangle. }\end{array}$ & $X$ & $X$ & \\
\hline $\begin{array}{l}\text { Promover y gestionar a la Secretaria del Medio } \\
\text { Ambiente y Recursos Naturales, acciones para la } \\
\text { vigilancia e inspección del manglar, además de } \\
\text { involucrar a la población en la conservación del mismo. }\end{array}$ & & $X$ & $X$ \\
\hline $\begin{array}{l}\text { Realizar recorridos eco-turísticos únicamente en } \\
\text { algunos puntos estratégicos, para no deteriorar el } \\
\text { área de manglar, lo cual pueda servir a los } \\
\text { pobladores para la obtención de recursos } \\
\text { económicos. }\end{array}$ & $X$ & $X$ & $X$ \\
\hline $\begin{array}{l}\text { Gestionar con el municipio alternativas para resolver } \\
\text { la problemática que existe sobre la colecta de basura. }\end{array}$ & $X$ & $X$ & \\
\hline $\begin{array}{l}\text { Llevar a cabo estudios sobre el control y calidad del } \\
\text { agua para prevenir la contaminación, la cual puede } \\
\text { afectar la biota acuática y terrestre del manglar. }\end{array}$ & $X$ & $X$ & $X$ \\
\hline
\end{tabular}

- Reconfiguración y Universidad Veracruzana. Xalapa, Ver. 120 p.

Contreras, F. y O. Castañeda. 1995. Los ecosistemas costeros del estado de Veracruz. Gobierno del estado de Veracruz. Secretaria de Desarrollo Agropecuario, Forestal y Pesquero. Veracruz, Veracruz, México. 144 p.

Fishdatabase. 2003. World wide web electronic publication.World Fish Center. EUA.

García-Hoyos, A.; L. Cadena; G. Carmona-Díaz y E. Rodríguez. 2003. Aprovechamiento del manglar de Sontecomapan en la reserva de biosfera Los Tuxtlas, Veracruz. Mesoamericana 7(1):46.

Hilton-Taylor, C. (Compiler). 2000. IUCN red list threatened species. I, Gland, Suiza y Cambridge, Reino Unido. $61 \mathrm{p}$.

Jiménez, J. 1999a. Ambiente, distribución y características estructurales en los manglares del pacífico de Centro América: Contrastes climáticos. In: A. Yáñez-Arancibia y A. L. LaraDomínguez, (eds) Ecosistemas de manglar en América Tropical. Instituto de Ecología, A.C., México, UICN/HORMA, Costa Rica, NOAA/ NMFS. Silver Spr MD. EUA. p:51-70. 
Jiménez, J. 1999b. El manejo de los manglares en el Pacífico de Centro América: Usos tradicionales y potenciales. In: A. Yáñez-Arancibia y A. L. Lara-Domínguez, (eds) Ecosistemas de manglar en América Tropical. Instituto de Ecología, A.C., México, UICN/HORMA, Costa Rica, NOAA/ NMFS. Silver Spr MD. EUA. p:275290.

Kjerve, B.; L. Drude y E. Hadji. 1997. Mangrove ecosystem studies in Latin America and Africa. UNESCO. Francia. 349 p.

Lacerda L., D. y Y. Schaeffer. 1999. Mangroves of Latin America: the need for conservation and sustainable utilization. In: A. YáñezArancibia y A. L. Lara-Domínguez, (eds) Ecosistemas de manglar en América Tropical. Instituto de Ecología, A.C., México, UICN/ HORMA, Costa Rica, NOAA/NMFS. Silver Spr MD. EUA. p:5-8.

Lahmann, E. 1999. La reserva forestal de Térraba-Sierpe, Costa Rica: Un ejemplo de uso adecuado del manglar. In: A. Yáñez-Arancibia y A. L. Lara-Domínguez, (eds) Ecosistemas de manglar en América Tropical. Instituto de Ecología, A.C., México, UICN/HORMA, Costa Rica, NOAA/NMFS. Silver Spr MD. EUA. p:291-298.

López-Portillo, J. y E. Ezcurra. 2002. Los manglares de México: una revisión. Madera y Bosques Número Especial 1:27-51.

Lugo, A. 2002. Conserving Latin American and Caribbean mangroves: issues and challenges. Madera y Bosques Número Especial 1:5-25.

Menéndez, L.F. 1976. Los manglares de la Laguna de Sontecomapan, Los
Tuxtlas, Veracruz. Estudio florísticoecológico. Tesis profesional. Facultad de Ciencias, UNAM. México, D.F. $40 \mathrm{p}$.

Morales-Abril, G. 1999. Guía para la elaboración de programas de manejo. Natural Conservancy. EUA. $78 \mathrm{p}$.

Morales-Mávil, J.E. y J. Villa-Cañedo. 1998. Notas sobre cacería y uso de fauna silvestre en Catemaco, Veracruz. Acta Zool. Mex. (n.s.) 73:127-144.

Paré, L.; E. Velásquez; R. Gutiérrez; F. Ramírez; A. Hernández; M. Lozada; H. Perales y J. Blanco. 1997. La reserva especial de la biosfera, Sierra de Santa Martha, Veracruz. Diagnóstico y perspectiva. SEMARNAP. PSSM. UNAM, IIS, Carlton University. $98 \mathrm{p}$.

Pennington, T.D. y J. Sarukhán. 1998. Manual para la identificación de campo de los principales árboles tropicales de México. Instituto Nacional de Investigaciones Forestales. Secretaría de Agricultura y Ganadería. Organización de las Naciones Unidas para la Agricultura y la Alimentación (FAO). México, D.F. y Roma, Italia. $115 \mathrm{p}$.

SEMARNAT (Secretaría de Medio Ambiente y Recursos Naturales). 2001. Norma oficial mexicana 059 que determina las especies y subespecies de flora y fauna silvestre terrestres o acuáticas en peligro de extinción, amenazadas, raras o las sujetas a protección especial y que establece especificaciones para su protección.

Silva-Benavides, A.M. 1999. Cuanti-ficación de la sociobiología del manglar de Purruja, Golfito: Recomenda- 
ciones para su manejo. Jornadas de investigación. Vicerrectoría de investigación. Universidad de Costa Rica. $67 \mathrm{p}$.

Tomlinson, P.B. 1986. The Botany of Mangroves. Cambridge University Press. Cambridge, Reino Unido. 98 p.

Valdez H., J.I. 2002. Aprovechamiento forestal de manglares en el estado de Nayarit, costa Pacífica de México. Madera y Bosques Número especial:129-145.

Villalobos, J.G.; A. Yáñez-Arancibia; J.W. Day y A.L. Lara-Domínguez. 1999. Ecología y manejo de los manglares en la Laguna de Términos,
Campeche, México. In: A. YáñezArancibia y A.L. Lara-Domínguez, (eds) Ecosistemas de manglar en América Tropical. Instituto de Ecología, A.C.. México, UICN/HORMA, Costa Rica, NOAA/NMFS. Silver Spr, MD. EUA. p:263-274.

Yáñez-Arancibia, A. y A.L. LaraDomínguez. 1999. Los manglares de América Latina en la encrucijada. In: A. Yáñez-Arancibia y A.L. LaraDomínguez, (eds) Ecosistemas de manglar en América Tropical. Instituto de Ecología, A.C., México, UICN/HORMA, Costa Rica, NOAA/NMFS. Silver Spr, MD. EUA. $\mathrm{p}: 9-16 . \diamond$ Sontecomapan, Catemaco, Veracruz, México: una estrategia para la conservación de sus recursos naturales. Madera y Bosques Número especial 2:5-23. 